\title{
Immunotoxin Cancer Immunotherapy
}

National Cancer Institute

\section{Source}

National Cancer Institute. Immunotoxin Cancer Immunotherapy. NCI Thesaurus. Code C15973.

Semisynthetic conjug ates of various toxic molecules, including radioactive isotopes and bacterial or plant toxins, with specific immune substances such as immunog lobulins, monoclonal antibodies, and antigens. The antitumor or antiviral immune substance carries the toxin to the tumor or infected cell where the toxin exerts its poisonous effect. 